\title{
Promoción de la salud de las personas con enfermedades raras y sus familias: experiencia de abordaje psicosocial
}

\section{Health promotion in people with rare diseases and their families: an experience of psychosocial approach}

\author{
Carmen Laborda López \\ Federación Española de Enfermedades Raras, España
}

\begin{abstract}
Resumen: En este trabajo se presenta la experiencia de atención desde un abordaje psicosocial a personas con enfermedades raras y sus familias, llevado a cabo desde el Servicio de Atención Psicológica y formación en coordinación con el Servicio de Información y Orientación de la Federación Española de Enfermedades Raras (FEDER). Esta experiencia forma parte de un programa de atención directa que tiene como objetivo mejorar la calidad de vida de las personas con una enfermedad rara y sus familias facilitando el acceso a información de calidad, así como acogiendo, apoyando y orientando a las personas que consultan. Además, se fomenta la creación e impulso de redes de personas afectadas, familiares y profesionales. Dentro de estos servicios la atención psicológica tiene como objetivo fomentar la normalización biopsicosocial y la inclusión de las personas y sus familias que afrontan la complejidad e impacto de convivir con el diagnóstico de una enfermedad rara.

Palabras clave: enfermedades raras, familia, abordaje psicosocial, atención directa, inclusión, red social, calidad de vida.

Abstract: This paper reports the experience with a plan to care for patients with rare diseases and their families based on a psychosocial approach and carried out by the Psychological Care Unit and the Information and Counseling Unit of the Spanish Federation of Rare Diseases. This experience is part of a direct care program intended to improve the quality of life of people with rare diseases and their families by helping them access useful information and giving them support and guidance. Implementation of networks formed by patients, families, and professionals is also promoted. The purpose of the psychological care provided in
\end{abstract}

La correspondencia sobre este artículo debe enviarse a los autores al e-mail: c.laborda@enfermedades-raras.org

(cc) BY-NC-ND Este es un artículo Open Access bajo la licencia CC BY-NC-ND. 
this project is to promote psychosocial normalization and inclusion of patients and families who face the complexity and burden of living with the diagnosis of a rare disease.

Keywords: rare diseases, family, psychosocial approach, direct care, inclusion, networks of people, quality of life.

Promoción de la salud de las personas con enfermedades raras y sus familias.

\section{Las enfermedades raras}

En la Unión Europea (UE) y sus estados miembros, España entre ellos, se denominan Enfermedades Raras (ER) o Enfermedades Poco Frecuentes (EPF) a aquellas enfermedades cuya prevalencia está por debajo de 5 por cada 10.000 habitantes. Son, por tanto, enfermedades que consideradas una a una, afectan a muy pocas personas, pero que, tomadas en su conjunto, implican a una gran parte de la ciudadanía y sus familias. Aproximadamente el $80 \%$ son de origen genético y dos tercios se manifiestan antes de cumplir los dos años de vida, hay que tener en cuenta su alto índice de morbilidad y mortalidad, su elevado grado de diversidad funcional y dependencia, su cronicidad, la mencionada complejidad etiológica y diagnóstica y que, en muchos casos, son potencialmente mortales, lo que permite la consideración de las personas afectadas como un colectivo con necesidades socio sanitarias específicas (Seco y Ruiz-Callado, 2016).

Las ER están caracterizadas por el gran número y la amplia diversidad de desórdenes y síntomas que varían no sólo de enfermedad a enfermedad, sino también dentro de la misma patología. La misma condición puede tener manifestaciones clínicas muy diferentes de una persona a otra. Hay, en efecto, una notable diversidad de subtipos de la misma enfermedad (Consejería de Sanidad. 2016).

Según datos de la Agencia Europea de Medicamentos (1999), hay entre 5.000 y 8.000 enfermedades raras distintas en la Unión Europea, que afectan al colectivo de pacientes tanto en sus capacidades físicas, como en sus habilidades mentales. El verdadero reto de la investigación en estas patologías es avanzar en el descubrimiento de tratamientos y en la detección de nuevas enfermedades (Orphanet, 2012).

Entre los principales obstáculos de quienes tienen una enfermedad poco frecuente destaca el difícil acceso al diagnóstico correcto, según datos extraídos del estudio sobre necesidades sociosanitarias de las personas con ER en España (FEDER, 2017), el 50\% de los casos de personas con EPF han sufrido retraso del diagnóstico. Y el 20\% este retraso ha sido superior a los 10 años. Un porcentaje similar se ha demorado entre 4 y 9 años. Tras el diagnóstico la información es escasa y existe poco conocimiento científico, alto coste del cuidado y desigualdad en la accesibilidad al tratamiento (Carrión, Echandi, Bañón, y Pastor, 2015) Además, en muchos casos no existen protocolos de coordinación entre profesionales sanitarios, sociales y educativos y la poca investigación existente está fragmentada y es insuficiente. Todo esto se traduce en un gran impacto social y psicológico en quién tiene la ER en su familia y entorno cercano.

Según el estudio de necesidades sociales realizado por el Ministerio de Trabajo y Asuntos Sociales: "Enfermedades Raras: Situación y demandas socio sanitarias" (2017), los problemas sanitarios, derivados en principio de la enfermedad, no van aislados de los problemas sociales.

De ahí la importancia que tiene que un problema como este se aborde de una forma integral y en colaboración y coordinación con todos los organismos competentes. Una de las prioridades del colectivo fue la de la identificación de problemas específicos, y el contacto con personas con ER con el objetivo de generar redes y de encontrar los cauces más adecuados para una solución total o parcial.

Hace 20 años las enfermedades poco frecuentes eran unas completas desconocidas. Las familias se sentían invisibles en un sistema social que no conocía sus necesidades y, por ende, que no podía dar respuesta a su situación. Sin embargo, las familias y las personas decidieron cambiar esta realidad (Gol-Montserrat, y Pi-Corrales, 2018).

Así nace FEDER con la misión de unir a toda la comunidad de familias con ER en España, haciendo visibles sus necesidades comunes y proponiendo soluciones para mejorar su calidad de vida, representando sus intereses, defendiendo sus derechos y promoviendo mejoras concretas para lograr su plena inclusión social. 


\section{Servicio de información y orientación}

FEDER desde sus comienzos pone a disposición de cualquier persona su Servicio de Información y Orientación (SIO) avalada por European Organisation for Rare Diseases (EURORDIS) como Red Europea de Líneas de Ayuda en Enfermedades Raras y termómetro de la situación de las personas afectadas por ER en España. Se trata de un servicio gratuito que tiene como objetivo mejorar la calidad de vida de las personas con una EPF y sus familias. El servicio cuenta con la colaboración de un Comité Asesor especializado y técnicos con amplia experiencia para ayudar a todas las personas que necesiten orientación en EPF.

Desde el SIO se trabaja en diversas áreas, por un lado, su labor principal es la de facilitar el acceso a la información de calidad a las familias y profesionales, así como atender a la persona con ER y a los familiares como puerta de entrada y coordinándose con otros servicios de la organización y asociaciones miembro, así como con otras entidades y organizaciones externas, para ello promueve la búsqueda continuada de recursos y especialistas de referencia. Además, trabaja para fomentar la visibilidad y el sentimiento de pertenencia al colectivo y combatir la exclusión y fomenta la creación e impulso de redes de personas afectadas, familiares y profesionales. Por otro lado, desde el SIO se dota de información al OBSER (Observatorio de enfermedades raras) y se elaboran informes y estudios para que las administraciones puedan hacer una planificación sanitaria, educativa, laboral y social que ofrezcan respuestas a las ER (Carrión, y Mayoral, 2017).

Desde los inicios de la puesta en marcha del SIO se detectó la necesidad de atender el impacto psicoemocional ante los desafíos de convivir con la enfermedad, el duelo y la pérdida de autonomía, los cambios drásticos en la autoestima y el auto concepto, el carácter crónico y/o degenerativo, los cambios en las relaciones sociales, familiares, laborales, etc., que suponen para el afectado y la familia convivir con una enfermedad rara o situación de búsqueda de un diagnóstico. Este y un sin número de variables psicológicas se ven afectadas en la situación de enfermedad, retroalimentando negativamente el estado de salud de la persona.

Así se hace incuestionable que las consecuencias de tener una enfermedad poco frecuente hacen que sea necesario el apoyo psicológico para facilitar el proceso de adaptación y disminuir el riesgo de consecuencias psicopatológicas.

La Organización Mundial de la Salud (OMS) (2013) incluye en el concepto de salud el "bienestar físico, mental y social”. Esta definición señala la importancia de los factores psicológicos y sociales en el proceso de salud-enfermedad y promueve una atención integral en la persona, con lo que, bajo esta definición, la atención y el apoyo psicológico y social se torna fundamental en el abordaje interdisciplinar de las personas con una enfermedad poco prevalente con el fin de mejorar su calidad de vida.

De esta manera la psicología toma un papel fundamental en el ámbito de las enfermedades raras, tanto a nivel de apoyo directo, como en el diseño de programas de formación e investigación clínica (Guitiérrez, 2016).

La Atención Psicológica, bajo esta premisa, ayuda a minimizar el impacto del duelo a través de la acogida, la escucha activa, la legitimación e identificación emocional y el empoderamiento del paciente y/o la familia en la toma de decisiones sobre sus propios procesos de salud.

En este abordaje se deben añadir las ventajas del soporte social a lo largo del tiempo y en las distintas etapas que van a sucederse desde el diagnostico. Encontrar personas que están viviendo la misma situación evita el aislamiento, produce mejoras en el estado de ánimo por no sentirte único, disminuye la ansiedad y el miedo, proporciona información sobre el manejo de dificultades, facilita estrategias de afrontamiento en base a otras experiencias, fomenta la cooperación, los valores solidarios, y la unión, e invita a la reflexión, promoviendo la responsabilidad y el empoderamiento en pro de nuestra salud y bienestar (Laborda, 2018).

Es por ello que las asociaciones de personas con ER así como FEDER como plataforma, trabajan como un gran recurso de soporte social, ya que, en cualquiera de sus formas, las asociaciones son el vehículo natural que tienen para realizar actividades que ayuden a alcanzar logros que por sí solos no conseguirían en la sociedad, lo que repercute a nivel biopsicosocial (Carrión, Echandi, Bañón, y Pastor, 2015).

En este contexto se enmarca el Servicio de Atención Psicológica (SAP) de FEDER y la red de psicólogos de ERs en España que tiene como objetivo promover una red de profesionales relacionados con el abordaje e intervención en EPF, así como fomentar las buenas prácticas y la formación desde los diferentes ámbitos de la psicología. Los estudios sobre la situación de necesidades sociosanitarias en ER de FEDER, así como en la 
Estrategia Nacional de 2009 y la revisión de 2014 (Ministerio de Sanidad. 2014), han considerado la formación de los profesionales y futuros profesionales como elemento necesario, tanto para una mejor comprensión de los problemas relacionados con las EPF así como para la búsqueda de soluciones compartidas (FEDER y CREER, 2009).

En un país caracterizado por la descentralización sanitaria, es nuestra responsabilidad como psicólogos tejer una red de profesionales fuerte, potente, formada, en la que todos los profesionales a nivel multidisciplinar aprendamos unos de otros para que el paciente, nuestro principal objetivo, se sienta respaldado.

Atención psicológica en enfermedades raras. Durante los últimos años, desde la psicología se apuesta por potenciar la resiliencia, entendida como aquella capacidad que tenemos las personas para afrontar las dificultades y adaptarnos a las adversidades para un buen manejo de esta nueva realidad. El desarrollo de la resiliencia protegerá a la familia de enfermar en términos psicológicos y reducirá también la vulnerabilidad en otros aspectos de su salud física (Grau Rubio, 2002). Para poder llegar a este nivel de empoderamiento de la persona y la familia, los profesionales de la psicología debemos de atender los procesos de duelo, aliviar el sufrimiento y acelerar el proceso natural de recuperación (Menudos Corazones, 2012).

Ante el diagnóstico de una ER se puedan experimentar situaciones de alto nivel de estrés, ansiedad, depresión y fatiga lo cual aumentará exponencialmente las probabilidades de deterioro de la enfermedad y de las repercusiones en el proceso de adaptación de convivir con esta (Ruíz, 2009). Trabajar en esta dirección y el conocimiento del propio proceso de enfermar y las estrategias utilizadas para afrontarla conlleva prevenir la evolución hacia un duelo patológico y el agravamiento de los síntomas, desarrollando acciones que eviten o disminuyan la pérdida de autonomía, favoreciendo el empoderamiento y la autorrealización y autoconfianza, y potenciando la espontaneidad y creatividad ofreciendo recursos en la solución de problemas y aceptación de hechos.

Como se detalla a continuación a través de un testimonio de una madre cuidadora de un niño diagnosticado de una enfermedad rara podemos observar la importancia de la acogida desde el servicio de información y orientación y el acompañamiento psicosocial desde una atención individualizada a través del servicio de atención psicológica, en esta ocasión con un doble objetivo, reducir el aislamiento y el sentimiento de soledad e impotencia y atender a la sobrecarga de la figura del cuidado y la desculpabilización en el propio autocuidado.

"En los momentos de mayor dolor e incertidumbre busqué ayuda porque veía mi barco naufragar. Llamé a FEDER y pregunté sin saber a dónde me conduciría ese camino. En FEDER me escucharon y me derivaron al Servicio de Atención Psicológica. Y empecé mi trabajo personal con una de las profesionales del servicio. Ella me AYUDÓ, y lo escribo en mayúsculas porque así lo siento, a pesar de que inconscientemente no se lo puse fácil. Nuestras sesiones fueron mi tabla de salvación. Gracias a su calidad humana, su profesionalidad y buen criterio me ayudó a conseguir algo que sola no habría podido alcanzar: aceptar la enfermedad de mi hijo y seguir caminando. No era consciente de la magnitud de mi bloqueo hasta que, conversando con ella en las sesiones, el nudo se fue deshaciendo. Ella me decía que tenía que cuidarme, 'pero cómo?', le contestaba muchas veces, 'si no tengo tiempo para nada', porque cuidar a un niño enfermo te consume, te aísla, te anula poco a poco. 'Si no te cuidas tú, no podrás ayudar a tu hijo, necesitas un tiempo para ti' contestaba, y tenía razón.

Y ahí estaba la clave, para poder ayudar a mi hijo tenía que ayudarme primero a mí misma, física-emocional y afectivamente, y en ello estoy. Los niños pequeños aprenden por imitación, somos sus modelos para lo bueno y para lo malo. Por consiguiente, si enseño a mi hijo con mi ejemplo que ante las dificultades me repongo, me levanto y sigo para adelante, él aprenderá a ser resiliente." (Mamá de un niño de 5 años con Esofaguitis Eosinofílica).

Bajo este modelo de atención hemos buscado dar respuesta a las necesidades individuales y familiares derivadas del proceso de la enfermedad trabajando con el objetivo de:

- amortiguar la experiencia de sufrimiento afectivo, relacional y social, prevenir y tratar las posibles alteraciones emocionales y comportamentales que pueden conllevar la enfermedad,

- apoyar en la adherencia al tratamiento y en los autocuidados,

- fortalecer la autonomía, la autoestima y la autoeficacia de la persona e impulsar sus fortalezas,

- favorecer el ajuste de sus expectativas vitales,

- promover sus relaciones sociales y mejorar la comunicación intrafamiliar teniendo en cuenta y valorando la situación del cuidador principal y prevenir/intervenir también en caso de sobrecarga de este. 
Hay que tener en cuenta, que en muchos casos las personas están sin diagnosticar o han vivido un largo proceso hasta conseguir el diagnóstico, carecen de tratamiento específico y de información adecuada. Estos procesos provocan en la persona sentimientos de incertidumbre con respecto a la evolución de la enfermedad que conllevan a un pronóstico incierto (FEDER y CREER, 2017).

De la misma forma, se generan consecuencias que agravan a nivel psicosocial la calidad de vida de la persona, algunos de los aspectos que más nos encontramos y que más impacto psicológico tiene son la incertidumbre ante los distintos aspectos relacionados con la enfermedad, el sentimiento de falta de control a nivel personal y a nivel social ya que la enfermedad obliga a modificar o a abandonar el rol que desempeña en su familia o en su trabajo en la mayoría de los casos, el rechazo y discriminación social, lo que conlleva una pérdida de la autoestima y aislamiento asociado al sentimiento de ser único, desamparo, desorientación e impotencia lo que genera cuadros de depresión, ansiedad, y sentimientos como miedo, angustia, rabia y culpa en un alto porcentaje de los casos.

Todas estas características suelen conllevar secuelas físicas, psicológicas, neurológicas, estéticas y afectivas, lo que atenta contra las relaciones familiares y sociales, contribuyendo a la aparición de problemas asociados (Ruíz, 2009).

La situación de enfermedad conlleva el afrontamiento de muchas situaciones difíciles a las que hay que adaptarse (los cambios en las rutinas diarias, las intervenciones quirúrgicas, el manejo del dolor (Rivas, 2018), los tratamientos, las crisis, la hospitalización, la relación con los profesionales de la sanidad, el manejo de emociones desagradables, etc.) por lo que la familia se ve obligada a entrar a una realidad nueva, desconocida, cambiante y agotadora que pone en riesgo su equilibrio físico y emocional.

La familia ante el diagnóstico. En el momento del diagnóstico la persona va a responder, a lo largo del tiempo, con una serie de reacciones emocionales o etapas (incertidumbre, desconcierto, oposición, rabia, tristeza y adaptación) que forman parte del proceso lógico de adaptación a la enfermedad (Neimeyer, 2002).

En función de cómo se movilicen estas emociones a lo largo de las sucesivas etapas se incrementará o disminuirá el sufrimiento asociado al impacto emocional, y se dificultará o facilitará el proceso de adaptación de la persona con una ER y de sus familiares a la nueva situación que tiene que afrontar. Por ello es importante que los diferentes profesionales de los equipos interdisciplinares podamos conocer y entender estas fases para así poder ofrecer el apoyo emocional adecuado en cada momento.

Cuando la persona afectada por una ER es un hijo/a, el impacto del diagnóstico es una experiencia traumática, la noticia es inesperada y está lejos de los planes de futuro imaginados por los progenitores. Independientemente de este caso, también existen otras circunstancias, dónde el diagnóstico puede recaer en otros miembros de la familia (progenitores) y en ese caso todos los miembros se ven sometidos a una resignificación de los roles familiares.

Las reacciones de cada familia ante el diagnóstico están influenciadas por muchas variables: la personalidad de sus miembros, la experiencia familiar con enfermedades, los sistemas de apoyo con los que cuenta, sus creencias religiosas, el nivel socioeconómico, el curso de la enfermedad y de su tratamiento y el grado de resiliencia familiar. Pero, en cualquier caso, el momento del diagnóstico es desestabilizador para cualquier familia, independientemente de su capacidad de adaptación a las situaciones de crisis (Grau Rubio, 2002).

Centrándonos en el caso de la noticia de una hija/o con un diagnóstico de una ER la primera reacción de los padres ante el diagnóstico será de una gran conmoción y se conoce como fase de desconcierto.

El momento del diagnóstico es vivenciado como un auténtico tsunami por la familia y a nivel emocional se caracteriza por una intensa respuesta de shock, incredulidad y aturdimiento. Estas emociones se intensifican al tratarse de una enfermedad de la que rara vez o nunca se ha tenido conocimiento. Si la enfermedad es hereditaria, y por lo tanto los familiares ya conocen el curso y evolución por otros miembros afectados, el desconcierto se incrementa, principalmente cuando se trata de diagnósticos severos. También si no se conoce tratamiento curativo y éste sirve únicamente de soporte o es sintomático.

En pocos minutos los progenitores deben asimilar gran cantidad de información acerca del diagnóstico y del tratamiento facilitado por los profesionales sanitarios. Y si bien son incapaces de recordar información médica durante los días posteriores al diagnóstico, difícilmente consiguen olvidar el momento en que fue informado del mismo ni al profesional del que recibieron la noticia (Anderson, Elliott, y Zurynski, 2013). 
Durante el proceso de diagnóstico y a nivel psicológico, además de las emociones desagradables que surgen durante el proceso de diagnóstico, la familia se ve obligada a realizar muchos cambios pues todos sus integrantes, en mayor o menor medida, tienen que aprender a convivir con la enfermedad rara, modificando su proyecto de vida y sus esquemas habituales de funcionamiento (Ruíz, 2009).

En definitiva, el proceso de cambio, reestructuración y aceptación que supone el diagnóstico de una ER lleva consigo un trabajo arduo para la familia, el cual será más llevadero si todos - entorno cercano, profesionales y sociedad en general- tomamos la responsabilidad que nos corresponde y creamos un espacio de sensibilización, información, comprensión, consideración y respeto.

A continuación, una familia nos acerca la realidad vivida alrededor de la noticia de una enfermedad rara, y todo el proceso realizado a través de la atención individual donde se trabajó a nivel psicoemocional en la readaptación de su vivencia y la aceptación de las pérdidas asociadas al diagnóstico. En esta ocasión la atención de realizó durante 3 meses en sesiones quincenales con el objetivo de reducir el impacto del diagnóstico y acompañar el proceso de duelo caminando hacia la aceptación y la redefinición de su nueva etapa vital.

"Un diagnóstico es una línea de meta y una línea de salida. Es la meta de un periplo en soledad buscando respuestas y tocando a puertas que se cierran en nuestras narices. Es una meta de noches en vela, de preocupaciones, de lecturas sobre síntomas y causas, de dolor, de ver sufrir, de impotencia, de desesperación, de gastar hasta lo que no se tiene por llamar a otra puerta más. [...] Un diagnóstico también es la línea de salida de una carrera de fondo; la de aprender a vivir con ello. Pero aprender de verdad, con mayúsculas. Aprender a asumirlo y nombrarlo con normalidad y sin lágrimas en los ojos (se tarda, y mucho). Aprender y asumir que ese nombre o frase apenas darán a los demás una mínima pista de lo que pasa y vive nuestro hijo. Aprender a identificar cada uno de los matices de su patología y entenderlos, entenderle, cuando se desborda. Aprender que le juzgarán y nos juzgarán y que no debe importarnos. Aprender a asumir que no siempre le comprenderemos como debiera y que no siempre lo haremos bien, porque somos padres conscientes, pero no padres perfectos. Aprender, en definitiva, a vivirlo en positivo y a normalizarlo. Aprender a valorar las pequeñas cosas y también las grandes [...] Lucharemos contra ello, por nosotros, por nuestro hijo y por esta sociedad que necesita conocer y saber [...] Y después de todo el proceso vaciamos nuestras mochilas de eso que antes llamaríamos problemas y las llenamos de esperanza y coraje" (Anónimo testimonio familia enfermedades raras)

La atención grupal para las familias. La atención a las familias contribuye a aliviar la conmoción y caminar hacia la aceptación de la enfermedad, atendiendo el proceso emocional para conectar con los puntos clave; quién era, quién soy ahora, quién puedo ser y quién no voy a volver a ser. Los abordajes terapéuticos desde el ámbito de la atención psicosocial requieren complementar y coordinar los tratamientos y la atención sanitaria que se presta, así como poner el acento en su participación para trabajar en un enfoque de salud positiva (Kabat, 2016). Desde los niveles de atención bio-psico-social, bajo un modelo de intervención multidimensional centrado en la singularidad de la persona y de las familias, hay factores de cambio que ofrecen la posibilidad de crear un nuevo proyecto de vida y que comprende la redefinición personal y familiar del propio proyecto vital.

Con el objetivo de caminar hacia esta redefinición, se presenta como imprescindible que tanto los individuos como las familias sean acompañados para conocer sus necesidades, los apoyos disponibles, el desarrollo de las competencias, y el soporte social. A través de grupos de Ayuda Mutua y talleres grupales se trata de buscar soluciones nuevas y soporte en personas que sean capaces de entender en profundidad lo que comporta este problema; posibilita una comprensión más amplia mostrando las posibilidades reales que se tiene frente a este (Bermejo, 2003). Suponen una mejora psicológica al rebajar la ansiedad y mejorar el estado de ánimo a través del intercambio de experiencias, emociones y necesidades; favorecer la adquisición de estrategias de afrontamiento ante situaciones difíciles, así como potenciar el desarrollo de los aspectos socioafectivos (Mayoral y Ceballos, 2010).

Además de la vertiente sanitaria y la vivencia de la enfermedad en el ámbito personal y familiar, las ER implican también consecuencias en el ámbito social, en las relacionecon los otros miembros de la comunidad 
por ello las redes de pacientes proporcionan valiosísima información sobre cuidados y recursos sociales. Asimismo, es muy importante el apoyo en la recuperación de la salud. "Las asociaciones ayudan a que el paciente tenga una visión diferente de sí mismo y que pueda mejorar la situación a partir de las experiencias de otras personas que están viviendo su misma situación” (Ancochea, 2009).

"Hay que evitar el aislamiento social creando redes de paciente ya que es muy importante la comunicación de los problemas, las necesidades, los miembros del grupo aprenden que no están solos" (Ancochea, 2009). En este sentido, las entidades -asociaciones de pacientes y familiares, pero también fundaciones y federaciones de asociaciones- interpretan un papel fundamental porque pueden ayudar a estas personas y sus familias a adquirir conocimientos muy útiles, así como una perspectiva más positiva y sosegada para afrontar la enfermedad y sus síntomas o dotarles de ámbitos de "respiro", además, de reconocerse como el motor fundamental de los cambios sociales.

Hay que señalar que, si bien es cierto que en un primer momento la enfermedad es fuente de desestabilización familiar, también a largo plazo y con los apoyos necesarios puede convertirse en una oportunidad para que la familia pueda reconocer sus fortalezas, y desarrollar las propias potencialidades. Es lo que se conoce como resiliencia familiar, entendida como el proceso de adaptación y ajuste de la familia a las exigencias de la enfermedad que fortalece al individuo y a la familia en el transcurso del tiempo (Gómez, y Kotliarenco, 2010).

Una comunicación abierta, empática y desde el respeto por parte de los profesionales socio sanitarios puede contribuir al desarrollo de la resiliencia familiar.

Es fundamental para los progenitores que puedan recibir de los profesionales apoyo emocional, información y orientación para recuperar así la estabilidad y seguridad necesarias para el afrontamiento de la enfermedad (Servicio de Neonatología Hospital Universitario La Paz. 2017).

A continuación, las familias nos acercan con esta "Carta al mundo" su realidad, y cómo tras un acompañamiento y trabajo a través de un grupo de ayuda mutua se genera un espacio de expresión, normalización y empoderamiento. Las sesiones grupales se realizaron durante 6 meses y en paralelo se atendieron de manera individual antes y/o durante el desarrollo del grupo a cada una de las familias ya que en algunos casos se requiere una atención más individualizada, más profunda del proceso y, más adelante, la persona se empodera y encuentra soporte social a través del grupo.

Carta al mundo:

"Ellos son nuestro motivo, por ellos nos dibujamos mil sonrisas para aliviar su dolor y calmarlos, aunque la mayoría de las veces queramos salir corriendo, [...] son ellos los que cada día nos muestran el valor de lo sencillo, de lo auténtico, de lo esencial... el valor de una sonrisa real, de una mirada sincera, el valor de lo que es realmente importante, del amor...la llegada de nuestro hijo/a ha cambiado toda nuestras pirámide de prioridades...y nos ha conectado con el valor del tiempo, el valor de vivir el presente "instante a instante", el valor de la empatía, del "ponerse en el lugar del otro" [...] Comprende que necesitamos tu ayuda, que necesitamos tu voz, únete a nosotros para que cada día seamos más, comprende que me siento muy solo/a y que tu comprensión, empatía, tu mano activa, y una mirada cariñosa y honesta me acompaña, [...] y que tu ayuda es necesaria para mí y permitirá que yo ayude a otros, creando así redes de esperanza." (Carta escrita por padres y madres de un grupo de ayuda mutua desde el Servicio de Atención Psicológica de FEDER, 2018).

\section{Conclusiones}

Las reacciones emocionales asociadas a al diagnóstico de una enfermedad poco frecuente, pueden provocar alteraciones conductuales y psicopatologías que influyen de forma negativa sobre el proceso de adaptación a la enfermedad y la calidad de vida de la persona afectada y su familia. Conocer su procesamiento acerca de la propia enfermedad y, en su caso, modificar las estrategias utilizadas para afrontarla, evitará esta situación tan negativa y prevendrá consecuencias psicopatológicas futuras.

Cada persona es diferente y, en cierta medida, cambiante, fruto de la interacción de una herencia, una biografía única e irrepetible, un contexto socio-cultural concreto, de la situación económico-laboral, del tiempo 
de espera hasta el diagnóstico etc. Aprender a observar estos factores y comprender cómo se relacionan entre sí es indispensable para ayudar de modo válido.

La Atención Psicológica, bajo esta premisa, ayuda a minimizar el impacto del duelo a través de la acogida, la escucha activa, la legitimación e identificación emocional y el empoderamiento del paciente y/o la familia en la toma de decisiones sobre sus propios procesos de salud.

Así como hemos visto claro la importancia de una atención individual a la persona con una ER y a la familia ante el proceso de duelo por el diagnóstico de una ER, no podemos olvidar las ventajas del soporte social a lo largo del tiempo y en las distintas etapas que van a sucederse desde el diagnostico. Encontrar personas que están viviendo la misma situación evita el aislamiento, produce mejoras en el estado de ánimo por no sentirte único, disminuye la ansiedad y el miedo, proporciona información sobre el manejo de dificultades, facilita estrategias de afrontamiento en base a otras experiencias, fomenta la cooperación, los valores solidarios, y la unión, e invita a la reflexión, promoviendo la responsabilidad y el empoderamiento en pro de nuestra salud y bienestar. Es por ello que las asociaciones de personas con enfermedades raras así como la Federación Española de Enfermedades Raras (FEDER) como plataforma, trabajan como un gran recurso de soporte social, para personas con una ER y sus familiares, ya que, en cualquiera de sus formas, las asociaciones son el vehículo natural que tienen para realizar actividades que ayuden a alcanzar logros que por sí solos no conseguirían para sí mismos y ante y para la sociedad.

\section{Referencias}

Agencia Europea de Medicamentos. (1999). Enfermedades raras y medicamentos huérfanos, Luxemburgo: European Medicines Agency (EMEA).

Ancochea, A. (2009). El asociacionismo ayuda a mejorar la relación médico-paciente. Médicos y pacientes. Obtenido de http://www.medicosypacientes.com/articulo/el-asociacionismo-ayuda-mejorar-la-relaci\%C3\%B3n-m\%C3\%A9dico-paciente

Anderson, M., Elliott, E. J., y Zurynski, Y. A. (2013). Australian families living with rare disease: Experiences of diagnosis, health services use and needs for psychosocial support. Orphanet Journal of Rare Diseases, 8(1), 22. https://doi.org/10.1186/1750-1172-8-22

Bermejo, J. (2003). La relación de ayuda. Madrid, España: Los Camilos.

Carrión, J., Echandi, L., Bañón, A. y Pastor, E. (2015). FEDER, La fuerza del movimiento asociativo. Murcia, España: FEDER.

Carrión, J. y Mayoral, E. (2017). El trabajo social en las Enfermedades Raras. Enfoque práctico y teórico. Madrid, España: FEDER.

Consejería de Sanidad. (2016). Plan de Mejora de la Atención Sanitaria a Personas con Enfermedades Poco Frecuentes de la Comunidad de Madrid. 2016-2020. Madrid, España: Dirección General Coordinación de la Atención al Ciudadano y Humanización de la Asistencia Sanitaria.

Federación Española de Enfermedades Raras. (2017). III Conferencia EUROPLAN en el marco de la EU Joint Action RD-ACTION. Madrid, España: Autor.

Federación Española de Enfermedades Raras (FEDER) y Centro Estatal de Referencia de Atención a Personas con Enfermedades Raras y sus Familias (CREER) (2009). Estudio sobre situación de necesidades sociosanitarias de las personas con enfermedades raras en España. Estudio ENSERio. Madrid, España: Autor.

Federación Española de Enfermedades Raras (FEDER) y Centro Estatal de Referencia de Atención a Personas con Enfermedades Raras y sus Familias (CREER) (2017). Estudio sobre situación de necesidades sociosanitarias de las personas con enfermedades raras en España. Estudio ENSERio. Madrid, España: Autor.

Gol-Montserrat, J. y Pi-Corrales, G. (. (2018). Libro blanco de las Enfermedades Raras en España. Madrid, España: Fundación Gaspar Casal.

Gómez, E. y Kotliarenco, M. (2010). Resiliencia Familiar: un enfoque de investigación e intervención con familias multiproblemáticas. Revista de Psicología, 19, 103-132.

Grau Rubio, C. (2002). Impacto psicosocial del cáncer infantil en la familia [Número monográfico]. Educación, Desarrollo y Diversidad, 5(2), 67-87. 
Guitiérrez, A. (2016). Enfermedades Raras y Psicología: Un paso más hacia la esperanza. Obtenido de https:// www.psicomemorias.com/enfermedades-raras-psicologia-paso-mas-hacia-la-esperanza

Kabat, J. (2016). Vivir con plenitud las crisis. Cómo utilizar la sabiduría del cuerpo y de la mente para enfrentarnos al estrés, el dolor y la enfermedad. Barcelona, España: Kairós.

Laborda, C. (2018). Principales objetivos de la atención psicológica, Madrid, España: AELMHU.

Mayoral, E. y Ceballos, E. (2010). Guía técnica para grupos de afectados y asociaciones. Ámbito social y psicológico. Badajoz, España: FEDER.

Menudos Corazones. (2012). Primeros momentos tras la pérdida de un hijo. Madrid, España: Autor.

Ministerio de Sanidad. (2014). Estrategias en Enfermedades Raras del Sistema Nacional de Salud. Madrid, España: Servicios Sociales e Igualdad.

Ministerio de Trabajo y Asuntos Sociales. (2017). Enfermedades Raras: Situación y demandas socio sanitarias. Madrid, España: EUROPLAN III, FEDER.

Neimeyer, R. (2002). Aprender de la pérdida. Una guía para afrontar el duelo. Barcelona, España: Paidós.

Organización Mundial de la Salud (2013). Salud mental: un estado de bienestar. Obtenido de https://www.who. int/features/factfiles/mental health/es/

Orphanet. (2012). Orphanet: sobre las enfermedades raras. Recuperado de https://www.orpha.net/consor/cgibin/Education AboutRareDiseases.php?lng=ES

Rivas, M. (2018). Guía de manejo del dolor. Herramientas de autoayuda. Madrid, España: Fundación Grunenthal.

Ruíz, B. (2009). Guía de apoyo psicológico para Enfermedades Raras. Sevilla, España: FEDER.

Seco, O. y Ruiz-Callado, R. (2016). Las enfermedades raras en España. Un enfoque social. Prisma Social. 17. 373-395.

Servicio de Neonatología Hospital Universitario La Paz. (2017). Monografía FiCare La familia integrada en los cuidados. Madrid, España: Autor.

\section{Vínculos de interés:}

- Federación Española de Enfermedades raras https://www.enfermedades-raras.org/

- Portal sobre enfermedades raras y medicamentos huérfanos https://www.orpha.net/consor/cgi-bin/index. php?lng=ES

- Observatorio de enfermedades raras https://obser.enfermedades-raras.org/ 